\section{OCCUPATIONAL RISK FACTORS FOR PROSTATE CANCER: A CASE-CONTROL STUDY IN GUADELOUPE (FRENCH WEST INDIES)}

${ }^{1}$ Karène Morvan, ${ }^{1}$ Luc Multigner, 1,2Pascal Blanchet, 'Danièle Luce. 'INSERM U1085, Pointe À Pitre, Guadeloupe, France; ' ${ }^{2}$ Service d'Urologie, CHU de Guadeloupe, Pointe A Pitre, Guadeloupe, France

\subsection{6/oemed-2014-102362.312}

Objectives To study the associations between occupation, industry and prostate cancer risk in Guadeloupe, a high incidence area.

Method Incident cases of prostate cancer (707 cases) and 722 population controls were included. Information on lifetime occupational history and other potential risk factors was collected by interview. Logistic regression was used to estimate adjusted oddsratios (OR) and their 95\% confidence intervals (CI).

Results A significantly decreased risk was observed in farmers $(\mathrm{OR}=0.5$; CI $0.4-0.7)$, whereas marginally elevated ORs were found for farm workers, especially in sugarcane and banana farming. Banana plantation workers had been exposed to chlordecone, an estrogenic insecticide previously found to be associated with prostate cancer risk in this population. Significantly increased risks of prostate cancer were found in stock clerks $(\mathrm{OR}=2.7$; CI 1.0-7.2), fishermen $(\mathrm{OR}=2.0$; CI 1.0-4.0), mail distribution clerks $(\mathrm{OR}=7.7 ; \mathrm{CI} 1.7-34.4)$ and electricians employed for more than 20 years $(\mathrm{OR}=4.0$; CI $1.0-15.8)$, as well as in public administration $(\mathrm{OR}=1.8$; CI 1.2-2.9), retail trade $(\mathrm{OR}=2.6$; CI 1.1-6.0) and manufacture of food products $(\mathrm{OR}=2.0$; CI 1.1-3.9), particularly sugar $(\mathrm{OR}=13.2$; IC 1.6108). Non-significantly elevated ORs were also seen for construction workers and transport equipment operators.

Conclusions Although the overall findings suggest that occupational factors have only a limited role in prostate cancer aetiology, elevated risks of prostate cancer were found in several occupations or industries. Exposure to pesticides, solvents, traffic-related air pollution, low physical activity, whole-body vibration may explain some of these increased risks.

\section{THE RELATIONSHIP BETWEEN SOCIOECONOMIC POSITION, WORKING CONDITIONS AND SICKNESS ABSENCE IN A LIFE-COURSE PERSPECTIVE}

${ }^{1}$ Ingrid Sivesind Mehlum, ${ }^{1}$ Karina Corbett, ${ }^{2} J o n$ Michael Gran, ${ }^{1}$ Petter Kristensen. ${ }^{1}$ National Institute of Occupational Health, Oslo, Norway; ${ }^{2}$ Department of Biostatistics, University of Oslo, Oslo, Norway

\subsection{6/oemed-2014-102362.313}

Objectives Socioeconomic position (SEP) in childhood and in adulthood, and work environment factors are predictors of sickness absence (SA). Our objective was to examine the relationships between these factors in a life-course perspective, which has hardly been done previously.

Method Our study sample was all employed individuals who partook in the HUNT study and who were born between 1967 and $1976(\mathrm{~N}=4530)$. Outcome was the risk of at least one SA episode in 2009. Educational attainment (5 categories) served as indicator of adult SEP, whereas highest parental education level and father's average income during early childhood (0-6 years) were indicators of childhood SEP. Work factors were job control, physically demanding work and shift work. Risk ratios (RRs) were estimated using Poisson regression.

Results $29 \%$ of the women and $17 \%$ of the men had SA during follow-up. There was a strong gradient according to adult SEP for both genders. The age-adjusted RR for having an SA episode, comparing highest and lowest educational levels, was 2.83 for women and 3.85 for men. The RR was marginally weakened in women $(-4 \%)$ and strengthened in men $(+18 \%)$, after adjusting for childhood SEP (Model 2). Including all work factors in the model reduced the RRs by 20\% compared to Model 2 (RR 2.20 and 3.62, respectively), the largest impact for physically demanding work (15\% reduction in RR).

Conclusions There were strong social gradients in SA, partly mediated through work environment factors in a life-course perspective. We found gender differences that are difficult to explain.

\section{MESOTHELIOMA INCIDENCE AND OCCUPATION IN THE NORDIC COUNTRIES - A FOLLOW UP DURING FOUR DECADES}

${ }^{1}$ Nils Plato, ${ }^{2}$ an Ivar Martinsen, ${ }^{3}$ Par Sparren, ${ }^{3}$ Elisabete Weiderpass. ${ }^{1}$ Institute of Environmental Medicine, Karolinska Institute, Stockholm, Sweden; ${ }^{2}$ Cancer Registry of Norway, Oslo, Norway; ${ }^{3}$ Department of Medical Epidemiology and Biostatistics, Stockholm, Sweden

\subsection{6/oemed-2014-102362.314}

Objectives The purpose of this study was to study differences in incidence of malignant mesothelioma between the Nordic countries.

Method We used data from the ongoing Nordic Occupational Cancer Study (NOCCA). Occupational title by 3-digit level was obtained from the countries' population and housing censuses in 1960, 1970, 1980 and 1990. A job-exposure matrix (JEM) was developed, including 25 carcinogens with specific exposure levels for 283 occupations for years 1945 to 1994, using national exposure databases and expert assessments. All mesothelioma cases (ICD-7 158 for peritoneum and 162.2 for pleura) in the Nordic countries 1961-2005 were identified through linkages with national cancer registers. We calculated Standardised Incidence Ratios (SIR) of mesothelioma for 53 occupations/occupational categories for men and women and linked with the NOCCA JEM.

Results A total of 7899 persons were diagnosed with mesothelioma in the Nordic cohort from 1961 to 2005, of which 24.3\% were women. There was an increased significant SIR of mesothelioma among 15 of the 53 occupations/occupational categories for men, and for nine different occupations for women. The men's excessed risk was observed in typical male-dominated occupations, highest for plumbers (SIR 4.64, 95\% CI 4.09 to 5.24), with a total of 241 cases.

Conclusions We found great consistency among men between countries with occupations associated with asbestos exposure. For women, we found greater diversity between countries and risk assessment in occupations not associated with asbestos exposure. Unclear diagnosis of mesothelioma of the peritoneum and misclassification of occupation may be behind this.

\section{OCCUPATIONAL EXPOSURE AND STROKE - A CRITICAL REVIEW OF SHIFT WORK, AND WORK-RELATED PSYCHOSOCIAL RISK FACTORS}

${ }^{1}$ Kristina Jakobsson, ${ }^{2}$ Per Gustavsson. 'Division of Occupational and Environmental Medicine, Lund University, Lund, Sweden; Institute of Environmental Medicine, Karolinska Institute, Stockholm, Sweden

10.1136/oemed-2014-102362.315 